\title{
Securing the Communication of Medical Information Using Local Biometric Authentication and Commercial Wireless Links
}

\author{
Vladimir I. Ivanov' ${ }^{1}$, Paul L. Yu ${ }^{2}$, John S. Baras ${ }^{3}$ \\ 'University of Maryland, College Park, MD, USA, vivanov@umd.edu \\ ${ }^{2}$ US Army Research Laboratory, Adelphi, MD, USA, paul.yu@arl.army.mil \\ ${ }^{3}$ University of Maryland, College Park, MD, USA, baras@umd.edu
}

Medical information is extremely sensitive in nature - a compromise, such as eavesdropping or tampering by a malicious third party, may result in identity theft, incorrect diagnosis and treatment, and even death. It is, therefore, important to secure the transfer of medical information from its source, i.e., the patient, to the system that collects and records it, or its technology adoption will face a strong resistance from the users. We consider the scenario where a patient has a portable, wireless medical device that transfers the medical information to a remote server. We decompose this problem into two sub-problems and propose security solutions to each of them: (a) to secure the link between the patient and the portable device, and (b) to secure the link between the portable device and the network. Thus, we push the limits of the network security to the cutting edge: authenticating the user using their biometric information, authenticating the device to the network at the physical layer, and strengthening the security of the wireless link with a key exchange mechanism. The proposed authentication methods can be used for recording the readings of medical data in a central database and for accessing medical records in various settings.

\section{Keywords}

Authentication, biometrics, communication, cryptography, physical layer

\section{Introduction}

Medical information is extremely sensitive in nature - a compromise, such as eavesdropping or tampering by a malicious third party, may result in identity theft, incorrect diagnosis and treatment, and even death. The misuse of a stolen identity for receiving healthcare may result in unexpected bills and, still worse, doctors may make incorrect diagnoses and apply deleterious treatments based on data from the identity thief's medical history [1]. The application of wireless sensor networks to healthcare systems and their integration with the conventional wireless communication networks creates new opportunities, such as automatic collection of readings of medical sensors, and new challenges, with security and data confidentiality being critical ones [2]. One platform for building sensor networks for medical care, including hardware and software implementations, that bridges the gap between the existing sensor network systems and the requirements of healthcare is proposed in [3]. A shortcoming, however, is its lack of security, both as authentication and as data protection. Another framework for remote home healthcare using GSM/GPRS is proposed in [4], but it uses passwords for authentication, does not make any security analysis, and does not propose a solution for the security weakness of Bluetooth. Other architectures require public key infrastructures for authentication [5] or impose constraints on the clock accuracy/synchronization [6], either of which is undesirable. And finally, the use of asymmetric keys is particularly problematic in energy-constrained and computationally-limited systems.

An interesting architecture and implementation that address the specific challenges in securing a wireless medical sensor network is [7]. The authors propose using biometric (fingerprint) and medical (ECG) information for authentication and elliptic curve public-key cryptography for establishing symmetric shared keys for data protection. Transferring the user's biometric and medical information 
over a wireless network and storing them in each base station, however, are problematic because a security compromise may have serious consequences as these types of information cannot be revoked. On the other hand, since the biometric information cannot be assumed to be secret, an attacker who captures a mote and has user's biometrics can impersonate the mote to the base station since the mote authentication is essentially done with user's biometrics (the motes do not have public key pairs). Moreover, each base station has to store the biometric and medical information, used for authentication, of all users, which poses considerable challenges for the system update and scalability. In addition, using medical data (from the sensor) as a second-tier authentication is problematic because the monitored data often is pathological (i.e., revealing disease symptoms) and therefore it may lead to unacceptably high false reject rate, thus disabling the communication altogether. Finally, ECG is also sensitive to the body condition, and when it comes to using it for authentication, the technology is commercially immature and relatively inaccurate.

An important objective in securing such networks is using commercially available solutions and requiring as little modification of the communication infrastructure as possible because the additional cost of the security solution can be a major obstacle for its mass adoption and deployment.

We consider the scenario where a user, e.g., the patient, has a portable, wireless medical device that is able to transfer medical information to a network access point. We decompose this problem into two sub-problems and propose our solutions in turn: (a) to secure the link between the user and the portable device, and (b) to secure the link between the portable device and the network access point.

We take the general view that data confidentiality is ensured by using cryptography. The conventional approach is to employ a symmetric key with appropriate length as this method is fast and provides strong protection. However, encryption and decryption alone do not provide authentication, i.e., verification of the claim about the identity (of a human or a system). Authentication is a critical element in the security link because if done improperly, it may lead to data transfer from an unauthentic sender or to an unauthentic recipient.

In this paper, we consider the authentication problem at two interfaces: between the user and the portable device, and between the portable device and the network access point (or a remote server). Clearly, these two problems lie in different domains - the first is human-to-machine authentication, while the second is machine-to-machine authentication - and thus they require different methods. The novelty of our work is twofold: (1) by using biometric authentication, we are effectively "pushing" the boundary of the authentication not only from the network to the device, but all the way to the end user, and (2) with a recent breakthrough in the cryptographic technology, we can vastly improve previously insecure communication links between the device and the network.

\section{Authentication in Two Steps}

We propose to split the authentication of a user to a network access point in two steps (see Figure 1).

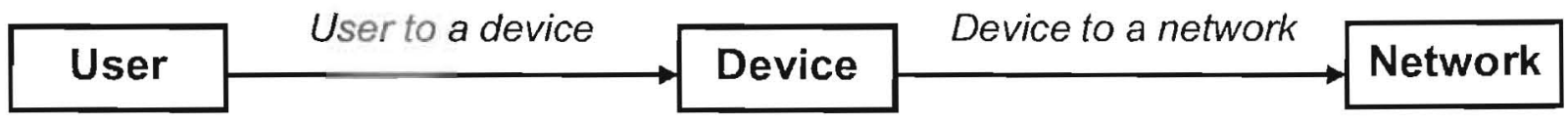

Figure 1 Authentication in two steps.

We use biometric information for the human-to-machine authentication. For the machine-to-machine authentication, we propose a novel, low-cost yet high-security approach based on the method of Markov key exchange [8].

\subsection{Local Biometric Authentication}

In the context of information technologies, biometrics is measuring, analysing, and using physiological and behavioural traits for identifying individuals. Biometrics has been used for automated authentication of people to systems for over a decade and makes the authentication more convenient because it does not require memorizing passwords or PIN codes. This convenience is particularly important in healthcare applications as the medical information may be time critical and require to be communicated even when the patient is under mental distress or physically unable to enter passwords or PIN codes. Furthermore, to be universally acceptable, a technology also has to be easy to use by 
people without specific technical training, in particular by seniors and children. Although easy to use, the RFID alternative and its derivatives cannot provide the required secure identification and authentication of humans because the RFID tags can be easily replaced (or their content changed) unless they are implantable, which, at this stage, is inapplicable because of numerous reasons (e.g., public acceptance, policy, health concerns, security weaknesses, and cost).

Today, many low-cost and small-sized systems for biometric authentication are commercially available and have the potential to become ubiquitous. Using biometrics for authentication, however, is problematic because the biometric information has a low degree of secrecy, i.e., it can easily be captured by an unintended recipient, which may occur even without the consent of the user [9]. The stolen information may be used to construct counterfeited or artificial biometrics, which has been shown to be relatively easy [10]. A compromise of individual's biometric information may lead to graver consequences than a compromise of a password. For example, in contrast to passwords, the biometric characteristics are not easily changeable and cannot be revoked, e.g., altering the person's fingerprint or iris cannot be done without surgical methods. In addition to the chronic security weaknesses of the common-place computer systems, function creep and owner abuse result in security breaches which are even harder to detect and thwart. Thus, storing the biometric information on a local computer, sending it over a network, and/or storing it on a remote server, even in encrypted form, would only further compound the problem. With over 260 million records, containing personal information such as Social Security numbers, account numbers, and driver's license numbers, compromised due to security breaches since January 2005 only in the US [11], the assumption that the biometric information can remain secret will be clearly wrong. Furthermore, a recent investigation by The Associated Press revealed that "banks and other companies that handle your information are not being nearly as cautious as they could," which results in "gambling with your personal data" once you pay with a credit card [12]. Under such circumstances, people's mistrust in the ability of systems and networks to protect their confidential information is completely justified.

We therefore propose to use biometric information to authenticate the user to a portable device, which device is user's personal property and in user's possession all of the time. We call this authentication local authentication. Thus, the biometric information is kept only in the device, not in a computer or a server on the network, and is locked onto the device. The locking is implemented using special hardware which ensures that the stored information cannot be compromised because the hardware inherently offers higher degree of security. The device essentially becomes "an extension" of the user and can be carried by the user at all time. Moreover, this approach requires little or no changes to the infrastructure, in particular, no modification of the security protocols for authentication of a device to a network. It also relaxes the expectations and assumptions about the trustworthiness of the user from the point of view of the network. And finally, the local authentication is capable of "hiding" the identity (e.g., the real name of the user) as it naturally shields the personal information from being sent over the network (or can instead use an identification number) without the need of additional network infrastructure, including a trusted third party, as proposed in [13].

Besides the purely technical arguments, the proposed method also helps gain the confidence of the users perceptually and psychologically. Users want to use a technology they are comfortable with but do not want to understand how it exactly works. For example, a patient knows that the biometric authentication works in other authentication scenarios, e.g., when appearing in person in a doctor's office. Now the patient is using her biometrics locally to authenticate to her doctor, which "brings" the doctor right "in front of her." Therefore, in addition to the technical guarantee about preserving the secrecy of the biometric information that our approach gives, it also makes the user more readily accept, and therefore take advantage of, the medical device, enabling the doctors and medical staff to provide better healthcare.

For biometrics, we propose the use of fingerprints. Human fingerprint patterns are highly distinct, develop early in life, and are relatively permanent [14]. Fingerprints have been used for identifying individuals for over a century. Initially systematized and developed for law enforcement, today fingerprints are widely used for access to facilities and for authentication to computer systems. Furthermore, low-cost and small-sized implementations of fingerprint sensors are available, making the authentication based on fingerprints particularly suited for portable devices.

Portable handheld devices have been increasingly used in a diverse set of applications - for communication (e.g., cell and smart phones), as personal data assistants (PDAs), and for access to financial services (e.g., hardware tokens). These devices have seen a long evolution in the access control to them: from not being protected at all to a password or a PIN code to the modern biometric authentication, with most ubiquitous being the fingerprint authentication $[15,16]$. 
A major challenge for the biometric authentication in our scenario (Figure 1) is that the authentication may take place in an unsupervised environment and at convenience of the user (e.g., at home). Thus, because the biometric information is not secret, an attacker who may have obtained the biometric information of the legitimate user will be able to provide it to the biometric sensor as a counterfeit. Another problem arises from the portability of the device - it may be easily stolen, giving the attacker physical access to the device and thus the ability to launch a powerful attack. Fortunately, recent work on detecting fake fingerprints [17] and detecting attacks on the fingerprint sensor have yielded to promising results [18].

To protect the confidentiality of the biometric information, we propose to use a Trusted Platform Module (TPM) [19], specified by the Trusted Computing Group (TCG). The TPM is incorporated in the portable device (see Figure 2) and protects the integrity and the confidentiality of data with hardware support. Thus, the biometric information is protected in the TPM or stored in the device, but encrypted with keys managed by the TPM, and never leaves the device. Our current research includes studying various methods for using the TPM to secure the storage of the user's biometrics. The TPM also identifies the device, performs integrity measurements and reports them via a mechanism called attestation. For example, the TPM can attest for the software running in the device and securely communicate this information to a remote server. Although a commercially available solution that combines a fingerprint authentication with a TPM to increase the security of a computer system is available [20], currently it uses a wired interface (USB) to connect to the computer and, because its design is proprietary, the mechanism for protecting the biometric information implemented in the device is not publicly available.

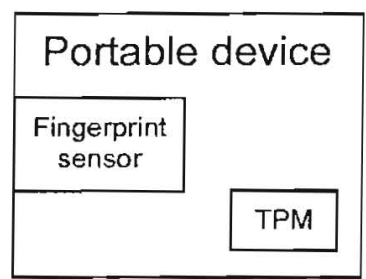

Figure 2 The portable device with a fingerprint sensor and a TPM.

The biometric information can be also securely stored into the smart chip of a smartcard [15]; the smart card is also a property of the user and is input into the portable device for performing the local authentication. Although the degree of tamper resistance of the smartcards is typically lower than that of the TPM, a smaricard provides greater flexibility as the biometric information can be stored only in one smartcard which is used in many portable devices.

We again stress the fact that it is this hardened security that encourages the use of the device. Very often new technologies are not adopted because users do not trust them and fear invasion of their privacy or theft of their private information.

\subsection{Authentication of a Portable Device to a Network Access Point or to a Remote Server}

Just as the user has to be authenticated and the portable device has to be secure, the communication from the device to a network access point or to a remote server has also to be secure. Also similarly to the biometric authentication, which uses inherent characteristics of humans, physical layer techniques, developed recently, exploit radio frequency characteristics to uniquely identify devices $[21,22]$. This techniques have been shown to offer high security while at the same time remain transparent to the existing communication technologies. Since this authentication is implicit to the device, the authentication adds to, rather than replaces, existing security measures. Thus, by combining our techniques with the currently used security methods, i.e., MAC and device authentication numbers, we significantly strengthen several aspects of the security of the device.

In the same vein of increasing security, we also demonstrate how another recently developed security measure can be used to strengthen the security of the wireless link $[23,24]$. Although we assume some type of key bootstrapping (e.g., via the initial key distribution), our method for key update is simpler, faster, and more efficient that the method proposed in [25].

The network access point needs to first ensure that the legitimate user is using the portable device. Therefore, no communication is permitted until the local biometric authentication is completed 
successfully. For example, if an attacker steals the portable device, no medical information will be transferred or requested until the genuine fingerprint is presented to the biometric sensor.

Let us now consider how we can harden (increase the security) of a commercially available wireless link for portable devices that has been proposed in pervasive healthcare applications, particularly in medical telemetry: Bluetooth [26]. This is a popular standard for low-cost devices that boasts low power and relatively high rate at short distances. However, its authentication protocol has a serious flaw and is notoriously weak. The main problem stems from the random nonces that are transmitted without encryption in the "mutual authentication" phase of the pairing process, which can be defeated with a "known plaintext" attack. In order to thwart this attack, we propose to encrypt the nonces before they are sent. A Markov key exchange method allows this to be done securely because of its large keyspace, in contrast to the Bluetooth PIN which consists of only 4 digits.

The Markov key exchange method uses synchronized Markov models to direct the key exchange between two parties [23]. Assume that initially the portable device and the network access point (or the remote server) is paired by using a shared secret key. The general idea is that when two parties share the same model, they can exchange and replace keys very rapidly while remaining synchronized. However, an attacker without the correct model will be unable to find out which key is being used. Therefore, the ability to find the correct keys is unique to the authentic parties, and this ability is the basis of the algorithm below that performs a two-party authentication.

We now detail a simple way for modifying the Bluetooth security protocol to avoid such popular attacks. Suppose that there are two parties and that in our scenario, Alice denotes the portable device and Bob denotes the network access point. Assume that Alice and Bob have already agreed on a secret master key $\mathrm{K}_{\mathrm{m}}$.

Algorithm for Authentication Using Markov Model-Directed Key Exchange

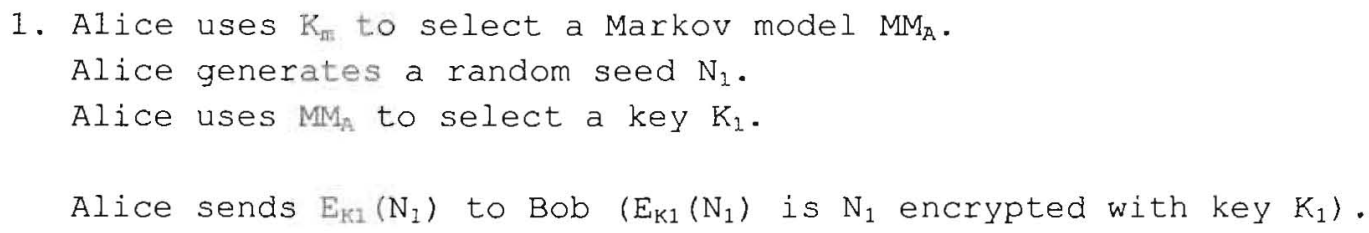

At this point, Bob can verify the authenticity of Alice because Bob is able to decrypt correctly the encrypted nonce $N_{1}$. This signals that Alice is using the same master key as Bob is to generate the keys. However, Alice does not know if Bob is authentic or not. Therefore, Alice needs to wait to see if Bob uses the correct key to encrypt the message that Bob will send.

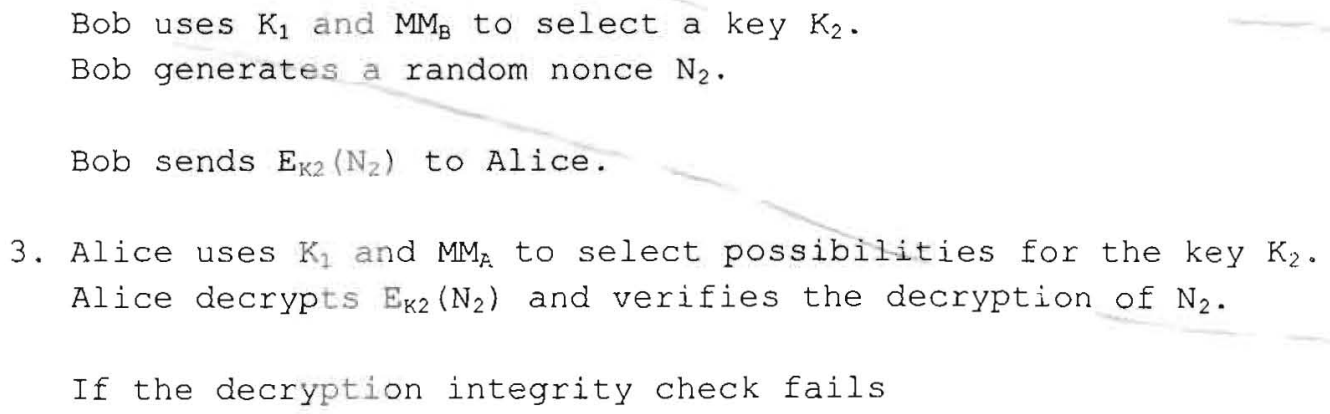


THEN halt. Since Alice is unable to decrypt $E_{K 2}\left(N_{2}\right)$, Alice knows

that Bob is using a different $K_{m}$ and the authentication fails.

ELSE continue.

At this point, Alice can verify the authenticity of Bob because Alice is able to decrypt correctly the encrypted nonce $\mathrm{N}_{2}$. This signals that Bob is using the same master key $\mathrm{K}_{\mathrm{m}}$ as Alice is to generate the keys. Now both parties have authenticated each other and the secure communication can begin.

Once the handshaking is complete, the secret keys are periodically refreshed automatically to harden the link security [23, 24].

The timeline of the algorithm is shown in Table 1.

Table 1 Timeline of the Markov model-directed key exchange algorithm.

\begin{tabular}{cccc}
\hline Time & Alice & Communication & Bob \\
\hline & $K_{m}$ & $<-$ Shared $->>$ & $K_{m}$ \\
\hline 0 & Sends $E_{K_{1}(}\left(N_{1}\right)$ & $-\cdots->$ & Finds $K_{1}$ to verify \\
\hline 1 & & & Sends $E_{K 2}\left(N_{2}\right)$ \\
\hline 2 & & & \\
\hline 3 & Finds $\mathrm{K}_{2}$ to verify & & \\
\hline
\end{tabular}

\section{Use Cases}

In this section, we first summarize the main advantages of our approach and then suggest two groups of applications of the methods we propose.

Using biometrics for authentication to a portable device is easier than using passwords or PIN codes as users (patients) need not memorize anything, need not have computer skills, and can authenticate even when under mental distress or physically unable to type. The local authentication limits the spread of biometric information by "locking it" in the device and never sending it to a computer or over a network, which significantly reduces the risk of compromising the biometric data. Furthermore, the security of a simple device (such as a PDA) is much easier to ensure than that of a computer, which typically runs many and diverse applications in a general-purpose operating system. Physically possessing the portable device all of the time increases user's confidence in the control of their private information, which makes the technology adoption easier. Finally, the intermediating portable device naturally allows hiding user's personal information, including their real name, from ever being sent to the network, thus enabling the delivery of anonymous services. As for the device-to-network authentication and security, our approach does not require a public key infrastructure and relies on physical layer techniques that inherently provide higher security. The efficiency of the key exchange mechanism allows simple implementations.

Figure 1 shows the two steps of the authentication process. Once the authentication is successful, secure information exchange between the user and the remote server may begin. The confidentiality and integrity of the data being transferred from this moment onward can be ensured by using secure protocols of higher levels, e.g., SSL or IPsec. Depending on the functionality of the portable device and the particular applications, the user may, for example, enter and read textual or graphical information, type their username (in the system) and request data to be retrieved from a remote server. We, however, do not discuss such details as they are not directly related to the problem of securing the communication at low level, i.e., by using biometric information and physical layer techniques. Thus, the suggested use cases only conceptually illustrate the two groups of applications.

\subsection{Recording the Readings of Medical Sensors in a Central Database}

Suppose, for example, that a patient has a sensor for monitoring the blood pressure or the heart beat rate, which readings are sent over to a remote database server that records the data and provides it to a physician for determining a diagnosis. The data may need to be recorded over a long period of time (e.g., over a month) and collected at any time of the day, including overnight. The medical sensor sends the data (locally) to the portable device, from which the data is transferred to a network access point and then to a remote server. The portable device should be able to authenticate first to the network access point and then to a remote server and securely (i.e., using encryption) transfer the 
data to it. The first step is authenticating the user to the device, and after that - authenticating the device to the network access point.

The medical sensor can, for example, read the data, send it to the portable device, which in turn can store it in its local memory. After the user authenticates to the device, the data transmission to the network access point is authorized, and the data is sent to the network. In this scenario, the patient has full control over the time when the medical data is transferred. In another scenario, it may be required that the measured data is sent immediately, in which case the patient can authenticate to the device only once, in the beginning, and grant a permission that the future transmissions take place automatically, without performing additional biometric authentications every time a piece of data is ready to be sent.

An exemplar application can be the service-based architecture WASP (Wirelessly Accessible Sensor Populations) for pervasive monitoring of elderly, introduced in [27], where the proposed biometric authentication takes place in the Personal Mobile Hub (PMH) and the proposed methods for increasing the link security are applied between the PHM and the Wireless Sensor Hub. Similarly, another possibility is the system for wearable vital signs monitoring, proposed in [28]. And finally, the authentication to the mobile phone in the architectures of [29] and [30] can be biometrics, and since the communication between the medical sensors and the mobile phone is Bluetooth, our methods for increasing the link security are also applicable. Although we do not assume the presence of a mobile network infrastructure and the functionality it provides, our method for local authentication (and sensor readings) can also be a part of the architecture proposed in [5].

\subsection{Access to Medical Records in a Central Database}

The proposed methods for authentication can also be used in a small group of nurses and doctors in a medical practice to authenticate users to medical data storage devices such as hard disks. Some hard drives, with very high capacity yet physically small, are already equipped with TPMs, which makes the implementation of the proposed authentication methods straightforward. Thus, not only is the access to sensitive information controlled, but at the same time the users are authenticated. The data transferred to the storage devices may include the medical history, current medications, and current readings of the medical sensors on the patient, and can be displayed in an easy to read format for fast assessment and action. The method for authentication can also be used in the framework for access to electronic patient records ([31] and [32]).

Another example application is the "personal data records." In the conventional "electronic data records," entering and maintaining patient's data is not the responsibility of the patient, but of someone else, e.g., the insurance company or the doctor. In the "personal data records," the patient enters and maintains the data, and, therefore, the patient must authenticate to the central database and use a secure communication channel to transfer the data (Kaiser Permanente in the US offers a similar service to its members [33]).

\section{Conclusions}

The application of wireless sensor networks to healthcare systems and their integration with the conventional wireless communication networks creates new opportunities and poses new challenges. Because of the very high sensitivity of the medical information, it is important to secure the transfer of medical information from the patient to the system that records and collects it. We propose to split the authentication problem in two authentication steps - of the user to a portable device and of the device to a network access point (or a remote server).

We propose to use biometrics for the authentication of the user to the portable device. Fingerprints are particularly suited for replacing the traditional passwords and PIN codes and provide the convenience and ease of use that is needed in medical applications. To protect the confidentiality of the user's biometrics, the biometric information is locked down in the portable device using the TPM technology and thus never leaves the device. This essentially makes the device "an extension" of the user. For the authentication of the portable device to the network access point and increasing the security of a popular commercial wireless link, we propose to use a physical layer authentication and a Markov key exchange method.

The proposed methods for authentication can be used for recording the readings of medical information to a central database and for access to medical records, in particular for "personal data 
records." The concept of separating the device authentication and the user authentication is also very important for telemedicine. Other applications of the proposed methods, beyond the scope of healthcare, are personal financial/bank services and mobile commerce. As our immediate next step, we consider implementing the proposed methods in a prototype system. Once this is successful, we will study, develop, and incorporate the high-level applications, including the appropriate software and communication protocols, which will enable the suggested use cases and allow real trials, i.e., connecting users (patients) with (healthcare) systems.

\section{References}

[1] Hagberg J. Palm-vein biometrics help accurately ID patients. SC Magazine [Internet]. 2008 Jul 2 [cited 2009 Jul 6]. Available from: http://www.scmagazineus.com/Palm-vein-biometrics-helpaccurately-ID-patients/article/112054

[2] Stankovic J A, Cao Q, Doan T, Fang L, He Z, Kiran R, et al. Wireless sensor networks for in-home healthcare: potential and challenges. HCMDSS Workshop: High Confidence Medical Device Software and Systems Workshop; 2005 Jun 2-3; Philadelphia, PA, USA. p. 2-3.

[3] Shnayder V, Chen B, Lorincz K, Fulford-Jones T R F, Welsh M. Sensor networks for medical care. Proceedings of the 3rd International Conference on Embedded Networked Sensor Systems; 2005 Nov 2; San Diego, California, USA.

[4] Jasemian $Y$. Security and privacy in a wireless remote medical system for home healthcare purpose. Proceedings of the Pervasive Health Conference and Workshops; 2006 Nov 29-Dec 1; Innsbruck, Austria.

[5] Shanmugam M, Thiruvengadam S, Khurat A, Maglogiannis I. Enabling secure mobile access for electronic health care applications. Proceedings of the Pervasive Health Conference and Workshops; 2006 Nov 29-Dec 1; Innsbruck, Austria.

[6] Elmufti K, Weerasinghe D, Rajarajan M, Rakocevic V, Khan S. Timestamp authentication protocol for remote monitoring in eHealth. PervasiveHealth 2008: Proceedings of the 2 nd International Conference on Pervasive Computing Technologies for Healthcare; 2008 Jan 30Feb 1; Tampere, Finland. p. 73-6.

[7] Malasri K, Wang L. Design and implementation of a secure wireless mote-based medical sensor network. Proceedings of the 10th International Conference on Ubiquitous Computing; 2008 Sep 21-24; Seoul, Korea. p. 172-181.

[8] Yu P L. Physical layer authentication [Ph.D. dissertation]. College Park (MD): University of Maryland, College Park; 2008 August.

[9] Chaos Computer Club publishes fingerprints of Wolfgang Schäuble, the German Home Secretary. Heise Online [Internet]. 2008 Mar 31 [cited 2009 Jul 6]. Available from: http://www.heise.de/ english/newsticker/news/105728.

[10] Matsumoto T, Matsumoto $H$, Yamada K, Hoshino S. Impact of artificial "gummy" fingers on fingerprint systems. Proceedings of SPIE; 2002 Apr 19; 4677: 275-289.

[11]A Chronology of Data Breaches. Privacy Rights Clearinghouse [Internet]; c2005-2009 [cited 2009 Jun 29]. Available from: http://www.privacyrights.org/ar/ChronDataBreaches. htm

[12] Robertson J. Weak security enables credit card hacks. The Associated Press [Internet]; 2009 Jun 14 [cited 2009 Jul 6]. Available from: http://tech.yahoo.com/news/ap/20090615/ap_on_hi_te/ us_tec_shoppers_gamble

[13] Weerasinghe D, Elmufti K, Rajarajan M, Rakocevic V. Patient's privacy protection with anonymous access to medical services. PervasiveHealth 2008: Proceedings of the 2nd International Conference on Pervasive Computing Technologies for Healthcare; 2008 Jan 30Feb 1; Tampere, Finland. p. 127-30.

[14] Maltoni D, Maio D, Jain A K, Prabhakar S. Handbook of fingerprint recognition. Springer; 2005.

[15] National Institute of Standards and Technology. Study report on biometrics in e-authentication. National Institute of Standards and Technology: INCITS M1/06-0424; 2006 May 15; Gaithersburg, MD, USA.

[16] Jansen W, Daniellou R, Cilleros N. Fingerprint identification and mobile handheld devices: an overview and implementation. National Institute of Standards and Technology: NISTIR 7290, 2006 Mar; Gaithersburg, MD, USA.

[17] Tan B, Schuckers S. Liveness detection for fingerprint scanners based on the statistics of wavelet signal processing. CVPRW'06: Proceedings of the 2006 Conference on Computer Vision and Pattern Recognition Workshop; 2006 Jun 17-22; New York, NY, USA. 
[18] Ivanov V I, Baras J S. Methods for estimating the intrinsic pattern of fingerprint scanners and using it in biometric authentication and for cryptographic key generation. University of Maryland, College Park; Invention disclosure number: IS-2009-023. Patent pending.

[19]Trusted Platform Module (TPM) specification v 1.2. [Internet]. Available from: http:// www.trustedcomputinggroup.org/developers/trusted_platform_module/specifications

[20]Ultra Mobile Authentication Key (UMAK). Product description. Inaura Inc. [Internet]. Available from: http://www.inaura.com.

[21]Baras J S, Yu P L, Sadler B M. Wireless communication method and system for transmission authentication at the physical layer. University of Maryland, College Park; Invention disclosure number: IS-2007-079. Patent pending. 2007 August.

[22] Yu P L, Baras J S, Sadler B M. Physical layer authentication. IEEE Trans Inf Forensics Secur. 2008 March; 3(1): 38-51

[23] Baras J S, Yu P L, Sadler B M. Method and implementation for key generation and replacement using Markov models. University of Maryland, College Park; Invention disclosure number: IS2008-112. Patent pending. 2008 October.

[24] Yu P L, Baras J S, Sadler B M. Key exchange using Markov models. ACM Trans Inf Syst Secur. [submitted].

[25] MacDonald J A. Cellular authentication and key agreement for service providers. PervasiveHealth 2008: Proceedings of the 2nd International Conference on Pervasive Computing Technologies for Healthcare; 2008 Jan 30-Feb 1; Tampere, Finland. p. 69-72.

[26] Scarfone K, Padgette J. Guide to Bluetooth security. National Institute of Standards and Technology Special Publication 800-121; 2008 Sep 3. Available from: http://csrc.nist.gov/ publications/nistpubs/800-121/SP800-121.pdf.

[27] Atallah L, Lo B, Guang-Zhong Y, Siegemund F. Wirelessly accessible sensor populations (WASP) for elderly care monitoring. PervasiveHealth 2008: Proceedings of the 2nd International Conference on Pervasive Computing Technologies for Healthcare; 2008 Jan 30-Feb 1; Tampere, Finland. p. 2-7.

[28] Chen W, Wei D, Zhu X, Uchida M, Ding S, Cohen M, Tokinoya S, Takeda N. A mobile phonebased wearable vital signs monitoring system. CIT 2005: Proceedings of the 5th Conference on Computer and Information Technology; 2005 Sep 21-23. p. 950-5.

[29] Rasid M F A, Woodward B. Bluetooth telemedicine processor for multichannel biomedical signal transmission via mobile cellular networks. IEEE Trans Inf Technol Biomed. 2005 March; 9(1): 35-43.

[30] Kailanto H, Hyvärinen E. Hyttinen J. Mobile ECG measurement and analysis system using mobile phone as the base station. PervasiveHealth 2008: Proceedings of the 2 nd international conference on Pervasive Computing Technologies for Healthcare; 2008 Jan 30-Feb 1; Tampere, Finland. p. $12-4$.

[31] Ferreira A, Barreto L, Brandao P, Correia R, Sargento S, Antunes L. A secure wireless architecture to access a virtual electronic patient record. Proceedings of the Pervasive Health Conference and Workshops; 2006 Nov 29-Dec 1; Innsbruck, Austria.

[32] Butz A, Kruger A. User-centered development of a pervasive healthcare application. Proceedings of the Pervasive Health Conference and Workshops; 2006 Nov 29-Dec 1; Innsbruck, Austria.

[33] Three Million People Now Using Kaiser Permanente's Personal Health Record. HealthcarelTNews [Internet]. 2009 Apr 22 [cited 2009 Jul 6]. Available from: http://www.healthcareitnews.com/pressrelease/three-million-people-now-using-kaiser-permanentes-personal-health-record. 